\title{
INDUÇÃO DA EXPRESSÃO PRECOCE DE SINTOMAS DE Guignardia citricarpa EM FRUTOS DE LARANJEIRA 'PÊRA-RIO'
}

\author{
RICARDO BRAGA BALDASSARI ${ }^{2}$, IVAN BRANDIMARTE ${ }^{3}$, ANDRÉ GUSTAVO DE ANDRADE ${ }^{4}$, \\ DANILO CESTARI GONÇALVES DE SOUZA ${ }^{5}$, CRISTIANE MORETTO ${ }^{6}$, ANTONIO DE GOES ${ }^{7}$
}

RESUMO - O presente trabalho teve por objetivo avaliar a influência de diferentes concentrações de ethephon na expressão precoce de sintomas de Guignardia citricarpa em frutos de laranjeira 'Pêra-Rio'. Para tal, frutos assintomáticos e isentos de aplicações com fungicidas, com 20 e 28 semanas após a queda de pétalas, foram coletados em área de comprovada existência da doença, no município de Conchal-SP e levados ao Laboratório de Fitopatologia da FCAV/UNESP, em Jaboticabal-SP, onde foram tratados com soluções nas seguintes doses de ethephon: i) 1,57 $\mathrm{g} \mathrm{L}^{-1}$; ii) 2,10 $\mathrm{g} \mathrm{L}^{-1}$; iii) 2,42 $\mathrm{g} \mathrm{L}^{-1}$; iv) Testemunha (água). Todas acrescidas de imazalil a $0,25 \mathrm{~g} \mathrm{~L}^{-1}$, para prevenir podridões de pós-colheita. Após os tratamentos, os frutos foram mantidos em câmara incubadora para B.O.D., calibrada à temperatura de $25^{\circ} \mathrm{C} \pm 1^{\circ} \mathrm{C}$, por 15 dias. Posteriormente, os frutos foram submetidos a quatro avaliações, em intervalos semanais, sendo atribuídas notas que variaram de zero (ausência de sintomas) a 6 (sintomas severos). Os dados da severidade da doença observados nos frutos colhidos prematuramente e submetidos aos diferentes tratamentos com ethephon foram comparados aos observados em frutos ensacados e não ensacados, mantidos no campo até a maturação natural. Constatou-se maior equivalência de sintomas nos frutos com idade entre 20 e 28 semanas, quando estes foram tratados com $2,10 \mathrm{~g} \mathrm{~L}^{-1}$ de ethephon e avaliados entre $28 \mathrm{e}$ 35 dias. Concluiu-se que o emprego de ethephon, nestas condições, viabilizou a expressão precoce dos sintomas da mancha preta em frutos contendo infecções quiescentes de G. citricarpa, com antecedência de, pelo menos, 105 dias antes da colheita. Tal resultado constitui-se, portanto, em alternativa de grande aplicabilidade na detecção precoce de sintomas de mancha preta.

Termos para indexação: Citrus sinensis, Phyllosticta citricarpa, ethephon, etileno.

\section{INDUCTION OF THE PRECOCE EXPRESSION OF Guignardia citricarpa SYMPTOMS IN FRUITS OF PERA-RIO SWEET ORANGE}

\begin{abstract}
The aim of this study was to evaluate the influence of different ethephon rates in the precocity expression of symptoms of black spot caused by Guignardia citricarpa in fruits 'Pêra-Rio' sweet orange, in the orchard located in Conchal, SP. Asymptomatic fruits without fungicides applications and with 20 and 28 weeks after petal fall were collected and taken to the Laboratory of Plant Pathology of FCAV/UNESP, in Jaboticabal/SP, where they were treated with the following ethephon rates: i) $1.57 \mathrm{~g} \mathrm{~L}^{-1}$; ii) $2.10 \mathrm{~g} \mathrm{~L}^{-1}$; iii) $2.42 \mathrm{~g} \mathrm{~L}^{-1}$; iv) (water) (control). For all treatments, Imazalil at $0.25 \mathrm{~g} \mathrm{~L}^{-1}$ was added to prevent post harvest fungal disease. After the treatments, the fruit were kept in a chamber with a temperature of $25^{\circ} \mathrm{C} \pm 1{ }^{\circ} \mathrm{C}$, during 15 days. Therefore, the fruits were evaluated four times with weekly interval using a scale from 0 (absence of symptoms) to 6 (severe symptoms). The data of disease severity observed in fruits colleted prematurely, under different ethephon rates were compared to those fruits bagged and unbagged remained in orchard until natural maturation. Major symptoms equivalence was verified in fruits with age 20 and 28 weeks, when they were treated with 2.10 $\mathrm{g} \mathrm{L}^{-1}$ of ethephon, and evaluated between 28 and 35 days. It is concluded that the use of ethephon in this conditions may cause a precocious expression of symptoms of black spot in fruits with quiescent infections of G. citricarpa with 105 days before of harvest. These results are an alternative of great applicability to precocious detection of citrus black spot.
\end{abstract}

Index Terms: Citrus sinensis, Phyllosticta citricarpa, ethephon, ethylene.

\section{INTRODUÇÃO}

A mancha preta dos frutos cítricos (MPC), cujo agente causal é o fungo Guignardia citricarpa Kiely (anamorfo: Phyllosticta citricarpa (McAlp.) Van der Aa), tem proporcionado elevados prejuízos à citricultura dos países onde ocorre, reduzindo significativamente a produção devido à queda prematura de frutos. Além disso, deprecia os frutos comercialmente e, por tratar- se de doença quarentenária para países da União Européia e Estados Unidos, onde a tolerância de frutos com sintomas de MPC é zero, limita grandemente a possibilidade de exportação de frutos in natura.

O controle da MPC baseia-se, principalmente, no emprego de fungicidas que, dado ao longo período de suscetibilidade dos frutos, por no mínimo 24 semanas (Baldassari et al., 2006), há

(Trabalho 063-06). Recebido em: 11-05-2006. Aceito para publicação em 02-03-2007.

${ }^{2}$ Eng. Agrônomo, Doutor, Consultor em Citricultura, Araraquara-SP .ricardo.baldassari@terra.com.br

${ }^{3}$ Eng. Agrônomo, Depto. Técnico Fundecitrus, Araraquara-SP.

${ }^{4}$ Eng. Agrônomo, Doutorando, UNESP-Jaboticabal, Depto. de Fitossanidade.

${ }_{5}^{5}$ Acadêmico em Agronomia, UNESP-Jaboticabal.

${ }^{6}$ Bióloga, Acadêmica de Mestrado, UNESP-Jaboticabal, Depto. de Fitossanidade.

Docente da UNESP-Jaboticabal, Depto. de Fitossanidade, CEP 14884-900, Jaboticabal/SP. agoes@fcav.unesp.br - (16) 3209-2640 
necessidade de várias pulverizações, elevando significativamente o custo de controle, que, atualmente, está estimado em cerca de $10,5 \%$ a 13,2\% do custo de uma caixa de laranja (Agrianual, 2005).

O uso de fungicidas sistêmicos, mesostêmicos e protetores, ou a combinação de ambos, constitui-se na principal medida de controle da doença, e suas respectivas concentrações e épocas de aplicação encontram-se bem definidas (Aguilar-Vildoso et al.,1999; Spósito et al., 1999; Feichtenberger et al., 2000; Reis et al., 2003; Feichtenberger \& Spósito, 2003).

Existem na literatura vários sintomas associados à MPC (Herbert, 1989; Goes et al., 2000), e dentre eles a mancha sardenta aparece em fases mais adiantadas de maturação dos frutos, embora a infecção tenha ocorrido quando o fruto se encontrava ainda jovem. Esse tipo de sintoma faz com que frutos, ainda que assintomáticos, mas contendo infecções quiescentes, manifestem tais infecções típicas nas fases mais adiantadas de maturação, às vezes, no local de destino. Nesses casos, quando se trata de frutos exportados, a carga poderá ser rechaçada, advindo elevados prejuízos aos exportadores. Assim, para o caso de frutos produzidos em áreas de ocorrência da doença e, mesmo sob criterioso programa de controle do patógeno e adoção de cuidados rigorosos nas inspeções no packing house, não se descarta a possibilidade do aparecimento de frutos sintomáticos no local de destino. Dessa forma, no caso de frutos destinados à exportação, oriundos de pomares existentes em regiões não indenes, há a necessidade de uma série de cuidados, incluindo-se o uso diferenciado de tratamentos químicos, combinado com uma série de práticas culturais. Além desses cuidados, há ainda a necessidade da realização de medidas adicionais que ampliem o nível de confiança dos exportadores.

Estudos prévios demonstraram que a aplicação de etileno antecipa a maturação dos frutos e pode ser uma alternativa com vistas à indução precoce dos sintomas da MPC (Bellotte et al., 2001).

Em frutos climatéricos, o etileno antecipa, acelera e uniformiza a sua maturação (Abeles et al., 1992; Goldschmidt, 1997), razão pela qual sua utilização é comum para esses tipos de frutos (Chitarra \& Chitarra, 1990). O etileno promove o aumento na atividade das enzimas clorofilase e oxidases (Shimokawa et al., 1978; Yamauchi et al., 1997), responsáveis pela degradação da clorofila e o conseqüente desaparecimento da cor verde, estimulando a carotenogênese, que promove o aparecimento das cores amarela ou laranja (Stewart \& Wheaton, 1972). Os frutos cítricos são classificados como não climatéricos, uma vez que seu processo de maturação não está associado a trocas importantes na velocidade de respiração, nem na produção de etileno. No entanto, a aplicação desse hormônio sobre os frutos proporciona um efeito estético positivo sobre a coloração da epiderme, que passa de verde para amarela ou laranja. Esta particularidade é um fato já conhecido e utilizado há tempos, uma vez que é prática usual para os frutos cítricos destinados ao consumo in natura, principalmente à exportação. Essa técnica faz com que os frutos que apresentem casca de cor verde, atinjam tons alaranjados, padronizando toda a carga quanto ao aspecto visual.
A determinação de um método seguro de prognóstico das

infecções quiescentes de Guignardia citricarpa, em frutos ainda jovens, poderá redundar em aumento da garantia fitossanitária dos frutos cítricos a serem colhidos em pomares conduzidos com objetivo da sua exportação. Nesse contexto, a finalidade desse estudo foi avaliar a eficiência do emprego de etileno, sob a forma de ethephon (Ethrel ${ }^{\circledR}$, Bayer CropScience), em diferentes concentrações, na indução de sintomas de MPC, em frutos de laranjeira 'Pêra-Rio', em dois estádios de desenvolvimento.

\section{MATERIAL E MÉTODOS}

Os ensaios de campo foram conduzidos em um pomar comercial de laranjeira 'Pêra-Rio' (Citrus sinensis L.), com 10 anos de idade, localizado no município de Conchal-SP, em área de comprovada existência da doença. As plantas destinadas ao experimento, bem como suas bordaduras, não receberam tratamentos com fungicidas, desde o florescimento até o completo desenvolvimento dos frutos. Já os procedimentos laboratoriais foram conduzidos no Departamento de Fitossanidade da UNESP, Câmpus de Jaboticabal.

O pomar utilizado para o experimento apresentava duas floradas distintas, uma ( $1^{\mathrm{a}}$ florada) ocorrida na segunda quinzena de setembro de 2002, e outra ( $2^{\mathrm{a}}$ florada), na segunda quinzena de novembro de 2002. Para cada tratamento e para cada uma destas floradas, uma amostra de 200 frutos, coletada de forma eqüitativamente distribuída por toda área, foi retirada no dia 6 de abril de 2006 e levada ao Laboratório de Fitossanidade da UNESPJaboticabal-SP, para serem submetidos a indução precoce dos sintomas de MPC. Assim, em relação à data de coleta, os frutos da primeira florada apresentavam-se com aproximadamente 28 semanas após a queda de pétalas e com diâmetro médio de $6,5 \mathrm{~cm}$. Já os frutos da segunda florada apresentavam-se com 20 semanas após a queda de pétalas e diâmetro médio de $5,0 \mathrm{~cm}$. Por ocasião da coleta destes frutos, procedeu-se também à identificação e ao ensacamento de um fruto localizado imediatamente ao lado de cada fruto retirado, utilizando-se de papel cristal de 18 × $8 \mathrm{~cm} . \mathrm{O}$ ensacamento foi realizado para evitar a continuidade da exposição natural dos frutos ao patógeno e, conseqüentemente, permitir verificar a ocorrência ou não de infecções posteriores, o que poderia influenciar no nível de severidade final da doença e inviabilizar as comparações pretendidas. Concomitantemente a esta coleta e ensacamento, outros 200 frutos adicionais, também próximos aos já coletados e aos ensacados, foram identificados e permaneceram expostos às infecções naturais, até o momento da colheita, que ocorreu em 04-08-2003, quando se encontravam maduros. No Laboratório, os frutos coletados em 6 de março de 2003 foram lavados e tratados, sob forma de imersão, por um minuto, nas seguintes concentrações $\left(\mathrm{g} \mathrm{L}^{-1}\right)$ de ethephon $\left(\right.$ Ethrel $^{\circledR}$, Bayer CropScience): i) 1,57; ii) 2,10; iii) 2,42; iv) testemunha (sem emprego de ethephon). Todos os tratamentos foram acrescidos de imazalil a $0,25 \mathrm{~g} \mathrm{~L}$, para prevenir podridões de pós-colheita, preservando os frutos para posterior análise da severidade da MPC. 
Após o tratamento, os frutos foram mantidos em câmara incubadora para B.O.D, calibrada à temperatura de $25^{\circ} \mathrm{C} \pm 1^{\circ} \mathrm{C}$, por 35 dias, com fotoperíodo 12/12 horas. A partir de 14 dias de incubação, tais frutos foram submetidos a quatro avaliações semanais, sendo empregada escala de notas, que variou de 0 a 6 , conforme metodologia utilizada por Fagan \& Goes (1999).

Em 4 de agosto de 2003, quando os frutos que permaneceram no campo, ensacados ou não, se encontravam maduros, procedeu-se a coleta dos mesmos, que também foram levados para o Laboratório de Fitopatologia para avaliação da severidade de G. citricarpa, empregando a mesma metodologia de escala de notas já mencionada. Embora existissem duas floradas distintas, a maturação dos frutos, de ambas as floradas, apresentou um padrão de maturação semelhante nessa data, o que não justificou colheitas em separado.

Os dados da severidade nos frutos colhidos precocemente e tratados com diferentes concentrações de ethephon em laboratório foram comparados àqueles verificados nos frutos de floradas correspondentes, ensacados e não ensacados (marcados), mantidos no campo. Para a análise de variância e comparação das médias, aplicou-se o teste de Tukey, ao nível de probabilidade de 5\%. Para facilitar as comparações, calculou-se o índice de correspondência entre as médias dos índices de doença, segundo fórmula proposta por Henderson \& Tilton (1955).

\section{RESULTADOS E DISCUSSÃO}

Os valores de severidade da doença, medida em índice de doença (ID), foram maiores em frutos originados da primeira floração (Tabela 1), uma vez que estiveram expostos a um maior período de infecção do que os da segunda florada (Tabela 2). Adicionalmente, quando se analisam os índices de infecção dos frutos mantidos a campo, com e sem ensacamento, observa-se que não houve um incremento expressivo nos índices de infecção nos frutos expostos às infecções naturais, após 28 semanas da queda das pétalas, com indicação, portanto, de que a maior suscetibilidade dos frutos está restrita a idades inferiores ao tempo mencionado. Já para os frutos expostos às infecções naturais após 20 semanas de queda de pétalas (segunda florada), ocorreu um incremento significativo no índice de infecção dos frutos mantidos a campo, com ensacamento, em relação aos frutos ensacados. Tais resultados, portanto, convergem aos obtidos por Baldassari (2006), o qual ressalta que frutos de laranjas 'Valência' e 'Natal' mostraram-se suscetíveis por, pelo menos, 24 semanas após a queda das pétalas, existindo, portanto, infecções posteriores à $20^{\mathrm{a}}$ semana de florescimento.

Os dados correspondentes aos valores do índice de doença (ID), para os diferentes tratamentos, para frutos de primeira florada, em comparação com o ID obtido nos frutos que foram mantidos a campo, com e sem ensacamento, encontram-se apresentados na Tabela 1. Situação semelhante se aplica para os frutos de segunda florada, cujos dados se encontram apresentados na Tabela 2. Em ambos os casos, verificou-se que houve um incremento temporal nos níveis de severidade da doença, mostrando-se progressivamente mais elevados com o tempo de incubação, alcançando os níveis elevados nas avaliações após 28 e 35 dias de incubação. Para o caso de frutos da primeira florada, o incremento temporal nos níveis de severidade da doença, nos frutos tratados e submetidos à incubação e avaliação, as variações foram de $143 \%$ a $227 \%$, entre 14 e 35 dias (dados não apresentados). Para o caso da segunda florada, tais incrementos foram ainda mais elevados.

Em relação aos frutos mantidos a campo até o amadurecimento, com ensacamento, os frutos do primeiro florescimento (Tabela 1), tratados com ethephon na maior concentração $(2,42 \mathrm{~g} / \mathrm{L})$ e avaliados após 35 dias de incubação, expressaram maior quantidade de sintomas da doença, diferindo estatisticamente da testemunha. Tal tratamento, entretanto, não diferiu daqueles constituídos por concentrações contendo $1,57 \mathrm{~g}$ $\mathrm{L}^{-1}$ de ethephon, após 28 e 35 dias de incubação, assim como daquele contendo 2,10 $\mathrm{g} \mathrm{L}^{-1}$ de ethephon, avaliado após 35 dias de incubação. Os níveis de severidade observados na última avaliação (35 dias), com exceção do tratamento-testemunha, foram estatisticamente semelhantes àqueles observados nos frutos que foram mantidos no campo, até o momento da colheita, com ensacamento. Já em relação aos frutos mantidos a campo até o amadurecimento, sem ensacamento, apenas o tratamento de 2,42 $\mathrm{g} \mathrm{L}^{-1}$ de ethephon, avaliado após 35 dias de incubação, apresentou quantidade de sintomas estatisticamente iguais, diferindo dos demais tratamentos.

Em relação aos frutos mantidos a campo até o amadurecimento, com ensacamento, no caso de frutos da segunda florada (Tabela 2), observou-se que o nível de severidade na avaliação realizada aos 28 e 35 dias após emprego de $2,10 \mathrm{~g} \mathrm{~L}^{-1} \mathrm{de}$ ethephon e na testemunha, após 35 dias, diferiram estatisticamente dos demais tratamentos e apresentaram um ID mais elevado, sendo estatisticamente semelhante ao nível de severidade da doença observado nos frutos mantidos a campo, até o amadurecimento, com ensacamento. Já em relação aos frutos mantidos a campo até $\mathrm{o}$ amadurecimento, sem ensacamento, também no caso de frutos da segunda florada (Tabela 2), apenas o tratamento $2,10 \mathrm{~g} \mathrm{~L}^{-1} \mathrm{de}$ ethephon, avaliado após 35 dias, apresentou ID estatisticamente semelhante ao nível de severidade da doença, observada nos frutos mantidos a campo, sendo, porém, estatisticamente diferente a todos os demais. Ainda para frutos provenientes de segunda florada, em relação a frutos mantidos a campo, sem ensacamento, para todos os tratamentos avaliados no período de 14 a 28 dias de incubação, os valores de ID foram estatisticamente inferiores àqueles verificados nos frutos mantidos a campo. Particularmente no caso da concentração contendo $2,42 \mathrm{~g} \mathrm{~L}^{-1}$ de ethephon, em todas as avaliações, os valores de ID foram significativamente mais baixos que aqueles observados nos frutos mantidos a campo. Tais resultados constituem-se em indicações de que, para o caso de frutos mais jovens e menores, como no presente caso, concentrações de ethephon superiores a $2,10 \mathrm{~g} \mathrm{~L}^{-1}$ podem inibir a expressão de grande parte dos sintomas, podendo advir falsos negativos. Suspeita-se que, dadas as propriedades químicas do ethephon, é possível que, para o caso de frutos cítricos ainda muito verdes, o mesmo altere suas características fisiológicas, refletindo em atraso ou na repressão parcial dos sintomas de MPC.

A equivalência, em termos de proporcionalidade dos níveis 
de sintomas (IDs) observados em frutos colhidos e tratados com diferentes concentrações de ethephon, seguido de incubação por $14 ; 21 ; 28$ e 36 dias após os tratamentos, comparados em relação ao ID observado nos frutos mantidos a campo, com e sem ensacamentos, para frutos da primeira e segunda florada, encontra-se apresentada nas Tabelas 3 e 4, respectivamente.

Para o caso de frutos da primeira florada, os maiores valores relativos, quanto aos níveis de equivalência entre os índices de doenças observados nos frutos trazidos e avaliados em laboratório, e os mantidos e avaliados no campo, com e sem ensacamento, foram verificados mediante o emprego de ethephon a 1,57 e 2,10 $\mathrm{g} \mathrm{L}^{-1}$, após incubação em B.O.D., calibrada à temperatura de $25^{\circ} \mathrm{C} \pm 1^{\circ} \mathrm{C}$, com fotoperíodo $12 / 12$ horas, por $28 \mathrm{e}$ 35 dias, principalmente (Tabela 3 ). Os valores de ID para os frutos submetidos a estas condições de incubação foram consistentemente mais elevados que os demais tratamentos, independentemente da concentração avaliada, quando comparados aos níveis de severidade dos frutos mantidos a campo, com ensacamento. Entretanto, quando em comparação com frutos mantidos a campo, porém sem ensacamento, o maior valor de ID, em termos absolutos, foram observados no tratamento constituído pela concentração contendo $2,42 \mathrm{~g} \mathrm{~L}^{-1}$ de ethephon aos 35 dias.

Para o caso de frutos da segunda florada, os maiores valores relativos quanto aos níveis de equivalência entre os índices de doença observados nas avaliações em laboratório, e aqueles em frutos mantidos a campo, com ou sem ensacamento, foram obtidos no tratamento constituído por ethephon a $2,10 \mathrm{~g}$ $\mathrm{L}^{-1}$, avaliado após 35 dias de incubação, em ambiente de incubação, como anteriormente mencionado (Tabela 4).

Os resultados ora obtidos constituem-se em alternativas práticas de relevância aos sistemas voltados às exportações de frutos cítricos in natura, uma vez que possibilitam a realização de um prognóstico quanto à presença de infecções quiescentes de G. citricarpa, com antecedência de, pelo menos, 105 dias antes da colheita. $\mathrm{O}$ melhor índice de correspondência entre os valores de ID em frutos de laranja 'Pêra-Rio', colhidos e avaliados precocemente, comparados àqueles mantidos na planta, sob condições naturais, ensacados ou não, foi obtido mediante a imersão dos frutos em solução de ethephon na concentração de $2,10 \mathrm{~g} \mathrm{~L}^{-1}$, por um minuto, seguido de incubação em B.O.D. a $25^{\circ} \mathrm{C}$, fotoperíodo $12 / 12$ horas e avaliados após 28 e 35 dias.

TABELA 1 - Média do índice de doença (ID) obtida aos 14; 21; 28 e 35 dias de incubação a 25ㄷ e $12 / 12$ horas, nos frutos provenientes da primeira florada, colhidos precocemente e tratados por imersão com diferentes doses de ethephon, comparada com frutos de mesma florada, mantidos a campo até o amadurecimento, com e sem ensacamento.

FRUTOS PROVENIENTES DA PRIMEIRA FLORADA

\begin{tabular}{|c|c|c|c|c|c|}
\hline \multirow{3}{*}{$\begin{array}{l}\text { Dose de } \\
\text { ethephon } \\
\left(\mathrm{g} \mathrm{L}^{-1}\right)\end{array}$} & \multicolumn{4}{|c|}{ Frutos Tratados } & \multirow{7}{*}{$\begin{array}{c}\text { Frutos mantidos a campo, } \\
\text { até o amadurecimento, } \\
\text { com ensacamento }\end{array}$} \\
\hline & \multicolumn{4}{|c|}{ Média do ID obtido após tratamento aos: } & \\
\hline & 14 (dias) & 21 (dias) & 28 (dias) & 35 (dias) & \\
\hline 0,00 & $1,95 \mathrm{Aa}$ & $3,36 \mathrm{ABb}$ & $3,39 \mathrm{ABb}$ & $3,95 \mathrm{Ac}$ & \\
\hline 1,57 & $2,36 \mathrm{ABa}$ & $3,87 \mathrm{Bb}$ & $3,94 \mathrm{Ab}^{*}$ & $4,69 \mathrm{Bc}^{*}$ & \\
\hline 2,10 & $2,26 \mathrm{ABa}$ & $3,13 \mathrm{Ab}$ & $3,86 \mathrm{Ac}$ & $4,19 \mathrm{Abc}^{*}$ & \\
\hline 2,42 & $2,62 \mathrm{Ba}$ & $3,18 \mathrm{Aa}$ & $3,09 \mathrm{Ba}$ & $4,65 \mathrm{Bb}^{*}$ & \\
\hline \multirow{3}{*}{$\begin{array}{l}\text { Dose de } \\
\text { ethephon } \\
\left(\mathrm{g} \mathrm{L}^{-1}\right)\end{array}$} & \multicolumn{4}{|c|}{ Frutos Tratados } & \multirow{7}{*}{$\begin{array}{c}\text { Frutos mantidos a campo, } \\
\text { até o amadurecimento, } \\
\text { sem ensacamento }\end{array}$} \\
\hline & \multicolumn{4}{|c|}{ Média do ID obtido após tratamento aos: } & \\
\hline & 14 (dias) & 21 (dias) & 28 (dias) & 35 (dias) & \\
\hline 0,00 & $2,11 \mathrm{Aa}$ & $3,06 \mathrm{ABb}$ & $3,20 \mathrm{Ab}$ & $4,19 \mathrm{ABc}$ & \\
\hline 1,57 & $3,00 \mathrm{Ba}$ & $3,50 \mathrm{Ba}$ & $4,18 \mathrm{Bb}$ & $4,47 \mathrm{ABb}$ & \\
\hline 2,10 & $2,53 \mathrm{ABa}$ & $2,78 \mathrm{Aab}$ & $3,25 \mathrm{Ab}$ & $4,01 \mathrm{Ac}$ & \\
\hline 2,42 & $2,09 \mathrm{Aa}$ & $3,26 \mathrm{ABb}$ & $3,18 \mathrm{Ab}$ & $4,76 \mathrm{Bc}^{*}$ & \\
\hline
\end{tabular}

Médias seguidas pelas mesmas letras maiúsculas nas colunas e minúsculas nas linhas não diferem, estatisticamente entre si, (Tukey, $P$ e"0,05).

* Médias assinaladas com asterisco são estatisticamente iguais às médias dos níveis de severidade (ID) observados nos frutos mantidos a campo (Tukey, $P$ e"0,05). 
TABELA 2 - Média do índice de doença (ID) obtida aos 14, 21, 28 e 35 dias de incubação a $25^{\circ} \mathrm{C}$ e $12 / 12$ horas, nos frutos provenientes da segunda florada, colhidos precocemente e tratados por imersão com diferentes doses de ethephon, comparada com frutos de mesma florada, mantidos a campo até o amadurecimento, com e sem ensacamento.

FRUTOS PROVENIENTES DA SEGUNDA FLORADA

\begin{tabular}{|c|c|c|c|c|c|}
\hline \multirow{3}{*}{$\begin{array}{l}\text { Dose de } \\
\text { ethephon } \\
\left(\mathrm{g} \mathrm{L}^{-1}\right)\end{array}$} & \multicolumn{4}{|c|}{ Frutos Tratados } & \multirow{7}{*}{$\begin{array}{c}\text { Frutos mantidos a campo, } \\
\text { até amadurecimento, } \\
\text { com ensacamento }\end{array}$} \\
\hline & \multicolumn{4}{|c|}{ Média do ID obtido após tratamento aos: } & \\
\hline & 14(dias) & 21 (dias) & $28($ dias $)$ & 35 (dias) & \\
\hline & $0,19 \mathrm{Aa}$ & $0,36 \mathrm{Aa}$ & $0,38 \mathrm{Aa}$ & $1,40 \mathrm{ABb} *$ & \\
\hline 1,57 & $0,18 \mathrm{Aa}$ & $0,57 \mathrm{Ab}$ & $0,75 \mathrm{Abc}$ & $1,03 \mathrm{Bc}$ & \\
\hline 2,10 & $0,21 \mathrm{Aa}$ & $0,51 \mathrm{Aa}$ & $1,30 \mathrm{Bb}^{*}$ & $1,50 \mathrm{Ab}^{*}$ & \\
\hline 2,42 & $0,14 \mathrm{Aa}$ & $0,62 \mathrm{Ab}$ & $0,65 \mathrm{Ab}$ & $1,25 \mathrm{ABc}$ & \\
\hline \multirow{3}{*}{$\begin{array}{l}\text { Dose de } \\
\text { ethephon } \\
\left(\mathrm{g} \mathrm{L}^{-1}\right)\end{array}$} & \multicolumn{4}{|c|}{ Frutos Tratados } & Frutos mantidos a campo, \\
\hline & \multicolumn{4}{|c|}{ Média do ID obtido após tratamento aos: } & até amadurecimento, \\
\hline & 14 (dias) & 21 (dias) & 28 (dias) & 35 (dias) & sem ensacamento \\
\hline 0,00 & $0,28 \mathrm{Aa}$ & $0,52 \mathrm{Aa}$ & $0,58 \mathrm{Aa}$ & $1,12 \mathrm{Ab}$ & \\
\hline 1,57 & $0,28 \mathrm{Aa}$ & $0,48 \mathrm{Aa}$ & $0,63 \mathrm{Aa}$ & $1,38 \mathrm{Ab}$ & \\
\hline 2,10 & $0,55 \mathrm{Ba}$ & $0,94 \mathrm{Ba}$ & $1,48 \mathrm{Bb}$ & $2,24 \mathrm{Bc}^{*}$ & 2,34 \\
\hline 2,42 & $0,11 \mathrm{Aa}$ & $0,41 \mathrm{Aa}$ & $0,63 \mathrm{Aa}$ & $1,34 \mathrm{Ab}$ & \\
\hline
\end{tabular}

Médias seguidas pelas mesmas letras maiúsculas nas colunas e minúsculas nas linhas não diferem estatisticamente entre si (Tukey, $P$ e"0,05).

* Médias assinaladas com asterisco são estatisticamente iguais às médias dos níveis de severidade (ID) observados nos frutos mantidos a campo (Tukey,

$P$ e'0,05).

TABELA 3 - Índice de correspondência entre a média dos índices de doença (ID) obtidos aos 14;21;28 e 35 dias de incubação a $25^{\circ} \mathrm{C}$ e 12/12 horas, em frutos de laranja 'Pêra-Rio', provenientes da primeira florada, colhidos precocemente e tratados por imersão com diferentes doses de ethephon, comparado ao ID de frutos de mesma florada, mantidos a campo até o amadurecimento, com e sem ensacamento.

\section{FRUTOS PROVENIENTES DA PRIMEIRA FL ORADA}

\begin{tabular}{|c|c|c|c|c|c|}
\hline \multirow{3}{*}{$\begin{array}{l}\text { Dose de } \\
\text { ethephon } \\
\left(\mathrm{g} \mathrm{L}^{-1}\right)\end{array}$} & \multicolumn{4}{|c|}{ Frutos Tratados } & \multirow{7}{*}{$\begin{array}{l}\text { Frutos mantidos a campo } \\
(\%) \text {, com ensacamento, até o } \\
\text { amadurecimento }\end{array}$} \\
\hline & \multicolumn{4}{|c|}{$\begin{array}{l}{ }^{1} \text { Índice de correspondência dos valores de ID obtidos } \\
\text { após tratamento com concentrações de ethephon aos: }\end{array}$} & \\
\hline & 14 (dias) & 21 (dias) & 28 (dias) & 35 (dias) & \\
\hline 0,00 & 44,72 & 77,06 & 77,75 & 90,60 & \\
\hline 1,57 & 54,13 & 88,76 & 90,37 & 107,57 & \\
\hline 2,10 & 51,83 & 71,79 & 88,53 & 96,10 & \\
\hline 2,42 & 60,09 & 72,94 & 70,87 & 106,65 & \\
\hline \multirow{3}{*}{$\begin{array}{l}\text { Dose de } \\
\text { ethephon } \\
\left(\mathrm{g} \mathrm{L}^{-1}\right)\end{array}$} & \multicolumn{4}{|c|}{ Frutos Tratados } & \multirow{7}{*}{$\begin{array}{l}\text { Frutos mantidos a campo } \\
(\%) \text {, sem ensacamento, até o } \\
\text { amadurecimento }\end{array}$} \\
\hline & \multicolumn{4}{|c|}{$\begin{array}{l}{ }^{1} \text { Índice de correspondência dos valores de ID obtidos } \\
\text { após tratamento com concentrações de ethephon aos: }\end{array}$} & \\
\hline & 14 (dias) & 21 (dias) & 28 (dias) & 35 (dias) & \\
\hline 0,00 & 43,42 & 62,96 & 65,84 & 86,21 & \\
\hline 1,57 & 61,73 & 72,02 & 86,01 & 91,98 & \\
\hline 2,10 & 52,06 & 57,20 & 66,87 & 82,51 & \\
\hline 2,42 & 43,00 & 67,08 & 65,43 & 97,94 & \\
\hline
\end{tabular}

Valores calculados pela formula de Henderson \& Tilton (1955). 
TABELA 4 - Índice de correspondência entre a média dos índices de doença(ID) obtidos aos 14;21; 28 e 35 dias de incubação a $25^{\circ} \mathrm{C}$ e 12/12 horas, nos frutos provenientes da segunda florada, colhidos precocemente e tratados por imersão com diferentes doses de ethephon e o ID obtido nos frutos de mesma florada, mantidos a campo até o amadurecimento, com e sem ensacamento.

\section{FRUTOS PROVENIENTES DA SEGUNDA FLORADA}

\begin{tabular}{|c|c|c|c|c|c|}
\hline \multirow{3}{*}{$\begin{array}{l}\text { Dose de } \\
\text { ethephon } \\
\left(\mathrm{g} \mathrm{L}^{-1}\right)\end{array}$} & \multicolumn{4}{|c|}{ Frutos Tratados } & \multirow{7}{*}{$\begin{array}{c}\text { Frutos mantidos a campo, } \\
\text { até amadurecimento, } \\
\text { com ensacamento }\end{array}$} \\
\hline & \multicolumn{4}{|c|}{$\begin{array}{l}\text { T'Índice de correspondência dos valores de ID obtidos após } \\
\text { tratamento com concentrações de ethephon aos: }\end{array}$} & \\
\hline & 14 (dias) & 21 (dias) & 28 (dias) & 35 (dias) & \\
\hline 0,00 & 11,73 & 22,22 & 23,46 & 86,42 & \\
\hline 1,57 & 11,11 & 35,19 & 46,30 & 63,58 & \\
\hline 2,10 & 12,96 & 31,48 & 80,25 & 92,59 & \\
\hline 2,42 & 8,64 & 38,27 & 40,12 & 77,16 & \\
\hline \multirow{3}{*}{$\begin{array}{l}\text { Dose de } \\
\text { ethephon } \\
\left(\mathrm{g} \mathrm{L}^{-1}\right)\end{array}$} & \multicolumn{4}{|c|}{ Frutos Tratados } & \multirow{7}{*}{$\begin{array}{c}\text { Frutos mantidos a campo, } \\
\text { até amadurecimento, } \\
\text { sem ensacamento }\end{array}$} \\
\hline & \multicolumn{4}{|c|}{$\begin{array}{l}{ }^{1} \text { Índice de correspondência dos valores de ID obtidos após } \\
\text { tratamento com concentrações de ethephon aos: }\end{array}$} & \\
\hline & 14 (dias) & 21 (dias) & 28 (dias) & 35 (dias) & \\
\hline 0,00 & 11,97 & 22,22 & 24,79 & 47,86 & \\
\hline 1,57 & 11,97 & 20,50 & 26,92 & 58,97 & \\
\hline 2,10 & 23,50 & 40,17 & 63,25 & 95,73 & \\
\hline 2,42 & 4,70 & 17,52 & 26,92 & 57,26 & \\
\hline
\end{tabular}

${ }^{1}$ Valores calculados pela formula de Henderson \& Tilton (1955).

\section{CONCLUSÃO}

O tratamento de frutos assintomáticos de laranjeira 'PêraRio', com idades entre 20 e 28 semanas após o florescimento, mediante imersão em solução de $2,10 \mathrm{~g} \mathrm{~L}^{-1}$ de ethephon por um minuto e avaliados entre 28 e 35 dias após, induz à expressão precoce de sintomas de Guignardia citricarpa em proporções equivalentes àquelas observadas em frutos maduros, mantidos na árvore, possibilitando prognosticar a presença da doença e sua severidade, com antecedência de até 105 dias da colheita.

\section{REFERÊNCIAS}

ABELES, F.B.; MORGAN, P.W.; SALTVEIT, M.E. Ethylene in plant biology. $2^{\text {nd }}$ ed. San Diego: Academic Press, 1992. 414p.

AGRIANUAL 2005: anuário da agricultura brasileira. São Paulo: FNP, 2005. p. 287-291.

AGUILAR-VILDOSO, C.I; FEICHTENBERGER, E.; MORAES, M.R.; SPÓSITO, M.B.; SCHINOR, E.H. Avaliação de tratamentos fungicidas no controle da mancha preta (Guignardia citricarpa) em laranjeira 'Pêra' de diferentes idades. Summa Phytopathologica, Jaboticabal, v.25, n.1, p.51,1999.

BALDASSARI, R.B.; REIS, R.F.; GOES, A. de. Susceptibility of Fruits of the 'Valência' and 'Natal' Sweet Orange Varieties to Guignardia citricarpa and the Influence of the Coexistence of Healthy and Symptomatic Fruits. Fitopatologia Brasileira,
Brasilia, v.31, n.4, p.337-341, 2006.

BELLOTTE, J.A.M.; ANDRADE, A.G. de; CARVALHO, C.R.B.; BALDASSARI, R.B.; GOES, A. de. Desenvolvimento precoce de sintomas da mancha preta causada por Guignardia citricarpa em frutos de laranjeira 'Pêra-Rio' através do tratamento com ethephon. Fitopatologia Brasileira, Brasília, v.26, p.397S, 2001

CHITARRA, A.B.; CHITARRA, M.I.F. Pós-colheita de frutos e hortaliças: fisiologia e manuseio. Lavras: ESAL/FAEPE, 1990. $320 \mathrm{p}$.

FAGAN, C.; GOES, A. de. Efeito da severidade da mancha preta dos frutos cítricos causada por Guignardia citricarpa na queda de frutos de laranja 'Natal'. Fitopatologia Brasileira, Brasília, v.24, p.282s, 1999.

FEICHTENBERGER, E.; GOES, A. de; BALDASSARI, R.B.; AGULAR-VILDOSO, C. I.; SPÓSITO, M.B. Manual técnico sobre pinta preta. Araraquara: Fundo Paulista de Defesa da Citricultura, 2000. 7p. (Boletim Técnico).

FEICHTENBERGER, E.; SPÓSITO, M.B. Tratamentos com fungicidas no controle de mancha preta (Guignardia citricarpa) em laranjeiras 'Valência'. Fitopatologia Brasileira, Brasília, v.28, p. 310S, 2003.

GOES, A. de; BALDASSARI, R.B.;FEICHTENBERGER, E.; SPÓSITO, M.B.; AGUILAR-VILDOSO, C.I. Cracked spot, a new symptom of citrus black spot (Guignardia citricarpa) in Brazil. In: INTERNATIONAL SOCIETY OF Citriculture, 9., 2000, Orlando. Programs and Abstracts...p. 145. 
GOLDSCHMIDT, E.E. Ripening of Citrus and other nonclimacteric fruits: effects of ethylene and other plant hormones. In: INTERNATIONAL SYMPOSIUM ON PLANT BIOREGULATORS IN FRUIT PRODUCTION, 8., 1997, Valencia, Abstract... Valencia: I.S.H.S., 1997.

HERBERT, J.A. Citrus black spot. Nelspruit: Citrus and Subtropical Fruit Research Institute, 1989. (Citrus H, 30)

HENDERSON, C.F.; TILTON, E.W. Tests with acaricides against the brown wheat mite. Journal of Economic Entomology, Lanham, v.48, n.2, p.157-161, 1955.

REIS, R.F. dos; GOES, A. de; PEREIRA, G.T. Efeito da aplicação de oxicloreto de cobre em diferentes épocas no controle da mancha preta dos citros causada por Guignardia citricarpa. Summa Phytopathologica, Botucatu, v.29, n. 1, p.12-18, 2003.

SHIMOKAWA, K.; SHIMADA, S.; YAEO, K. Ethylene-enhanced chlorophylate activity during degreening of Citrus unshiu Marc. Scientia Horticulturae, Amsterdam, v.8, n.2, p.129$135,1978$.

SPÓSITO, M.B.; AGUILAR-VILDOSO, C.I.;MORAES. M. R.; FEICHTENBERGER, E.; RUBIM, C.A. Avaliação de tratamentos fungicidas da mancha preta em frutos de laranjeira 'Natal'. Fitopatologia Brasileira, Brasília, v.24, p. 334, 1999.

STEWART, I.; WHEATON, T. A. Carotenoids in citrus: their accumulation induced by ethylene. Journal of Agriculture and Food Chemistry, Washington, v.20, n.2, p.448-449, 1972.

YAMAUCHI, N.; AKIYAMA, Y. KAKO, S.; HASHINAGA, F. Chlorophyll degradation in Wase satsuma mandarin (Citrus unshiu Marc.) fruit with on-tree maturation and ethylene treatment. Scientia Horticulturae, Amsterdam, v.71, n.1/2, p.35-42, 1997. 